\title{
Atención a estudiante no vidente: Caso en la asignatura de Matemática I para I y II Ciclos
}

\author{
Estibaliz Odilie Rojas Quesada ${ }^{1}$. Rónald Sequeira Salazar ${ }^{2}$,
}

\author{
1. Universidad Estatal a Distancia, Sabanilla, Costa Rica. erojasq@uned.ac.cr
}

2. Universidad Estatal a Distancia., Sabanilla, Costa Rica. rsequeira@uned.ac.cr

Recibido: 10 de Setiembre de 2019 Aceptado: 16 de Diciembre de 2019

\section{RESUMEN}

En este artículo se presenta el uso de una plantilla que se asemeja al cuadro del valor posicional que se utiliza en las operaciones aritméticas. Una estudiante no vidente de la carrera Educación General Básica I y II Ciclos en la asignatura de Matemáticas I para I y II Ciclos de la Universidad Estatal a Distancia de Costa Rica (UNED), como parte de su trabajo académico tiene que desarrollar una mini-clase con estudiantes de cuarto grado de educación primaria con el tema de la multiplicación con números naturales, y utiliza esta plantilla como parte de la adecuación que se hizo en el uso la pizarra. Para asesorarla, se consultó con especialistas en la atención de este tipo de población y se visitó a la estudiante en su Centro Universitario donde se llevó a cabo la experiencia.

Palabras claves: Personas ciegas, estrategias pedagógicas, enseñanza de la matemática, docente en formación.

\section{ABSTRACT}

This article show the use of stencil similar a table of positional values used in the arithmetic operations. A blind student of the Basic General Education First and Second Cycles in the course of Mathematic I to I and II Cycles of the Distance State University of Costa Rica (UNED), as part of his academic task, she has to develop a mini-class with fourth grade of elementary students with the topic of product with naturals number, and she uses this stencil like a part of adequacy that was done to use the whiteboard. To advice her, was consulted a specialist in the attention of this kind of population and we visited the student in her University Center where she did do her job.

Key words: Blind people, pedagogical strategies, teaching of mathematics, teacher in training

\section{Introducción}

En la cátedra de Matemáticas Básicas se tiene la asignatura de Matemáticas I para I y II Ciclos, código 00809, que se oferta en la Universidad Estatal a Distancia de Costa Rica (UNED) para la carrera de Educación General Básica I y II Ciclos, la cual tiene como finalidad contribuir a que los futuros docentes adquieran los conocimientos básicos relacionados con: sistemas de numeración, números naturales, teoría de números racionales positivos, relaciones y álgebra, así como algunas estrategias metodológicas para la implementación en el aula de los contenidos matemáticos mencionados.

Los estudiantes de esta carrera como futuros docentes en educación primaria, en su campo de trabajo deberán aplicar estrategias metodológicas innovadoras para el abordaje de los temas matemáticos, de ahí entonces que esta cátedra estableció que para la asignatura Matemática I para I y II Ciclos, el estudiante deberá realizar una mini-clase como parte de sus tareas académicas, la cual consiste en diseñar e implementar una estrategia metodológica innovadora para ser aplicada entre 2 y 4 lecciones en los niveles de cuarto a sexto grado de educación primaria y utilizando los programas de estudios de Matemática del Ministerio de Educación Pública (MEP, 2012) y las orientaciones pedagógicas del MEP (2006) para la atención de este tipo de población.

Para el diseño de la estrategia metodológica, los estudiantes en el entorno virtual de la asignatura tienen a su disposición el Programa de Estudios de Matemática del MEP, una guía de cómo elaborar el planeamiento didáctico para la lección y un documento con ejemplos de estrategias metodológicas implementadas por otros docentes.

Es importante señalar, que esta carrera cuenta con una estudiante no vidente por lo que la UNED en el Departamento de Atención a Necesidades Educativas Especiales, le brinda apoyo académico con audiolibros, conversión de documentos en formato Word, PDF y 
otros, para su respectiva lectura con el Programa Joyce, calculadoras parlantes, aplicación de pruebas escritas en Braille y en un recinto aparte con un docente de educación especial relacionado con el área de la asignatura.

\section{Materiales y métodos}

En esta experiencia de aprendizaje se asesoró a la estudiante en cómo elaborar su estrategia metodológica con algunos de los contenidos propuestos por la cátedra y que se encuentran en el plan de estudios de Matemática del MEP. Se contó también con la asesoría de un tutor experto del Programa de Enseñanza de la Matemática, quien además de laborar para la UNED, es docente en el área de Matemática en el instituto Hellen Keller, ente especializado en pedagogía para la atención de la población no vidente en el país. Cabe señalar, que el experto sugirió trabajar en la estrategia metodológica el tema de fracciones utilizando legos, ya que por su experiencia con los estudiantes de la Hellen Keller, manifiesta que "este tipo de recurso didáctico es más fácil de manipular, lo que permite un mejor desempeño en un ambiente amigable con su realidad táctil y le facilita al docente las explicaciones que va a dirigir al estudiante" (Badilla, 2016).

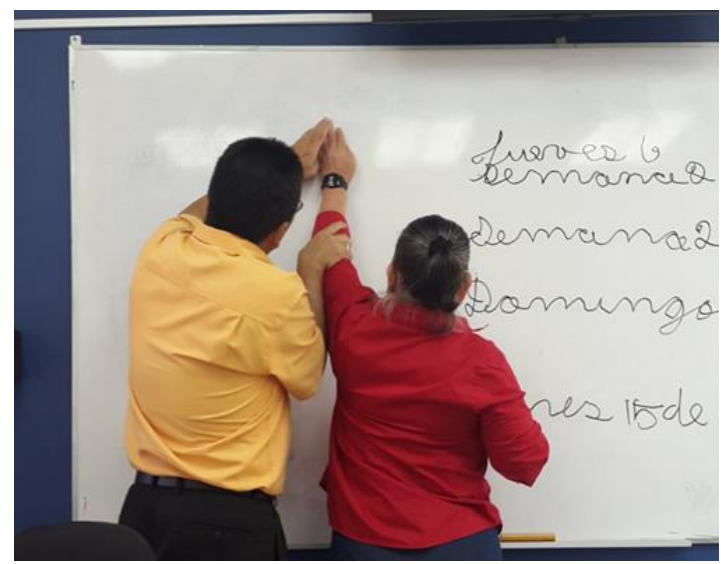

Figura 1. Reconocimiento del espacio para trabajar. Fuente: Fotografías tomadas el día de atención. Respaldos de la Cátedra de Matemáticas Básicas

Posteriormente, se le presenta a la estudiante el tema sugerido, pero se niega y manifiesta que no le interesa pues desea trabajar solamente con la multiplicación de números naturales. Esta situación es un claro ejemplo de la importancia de tener presente cuáles son los intereses y preferencias de los estudiantes sin importar su condición, pues como lo afirma Gutiérrez (2002) "una educación con sentido educa protagonistas, seres para los cuales todas y cada una de las actividades, todos y cada uno de los conceptos, todos y cada uno de los proyectos, significan algo para la vida".

Así entonces, dado que la estudiante no acepta la temática propuesta, se le contacta para diagnosticar in situ el dominio de contenidos matemáticos y su desempeño en la pizarra, pues este recurso didáctico es necesario para el desarrollo de los contenidos de la miniclase y en el trabajo que ella va a llevar a cabo con sus estudiantes en el salón de clase. Para determinar si la estudiante puede reconocer las dimensiones de la pizarra y si hay un buen uso del recurso, se le pide que escriba algunas palabras (Figura 1).

Dado de que no logra determinar de buena manera las dimensiones de la pizarra y que escribe "por todas partes", se comprueba que no sabe utilizar de forma correcta la pizarra, por lo que se le brindan una serie de sugerencias sobre su uso, pues cuando se trabaja con estudiantes de educación primaria, el buen uso de este recurso didáctico por parte del docente es de vital importancia, si se toma en cuenta que son alumnos que están en sus primeros años de formación. También, se le solicita que realice una multiplicación y se observa que tiene problemas de ubicación de los numerales, pues al no tener el sentido de la vista tiene que recordar las cifras que debe sumar y el espacio en el que debe ubicarlas (Figura 2).

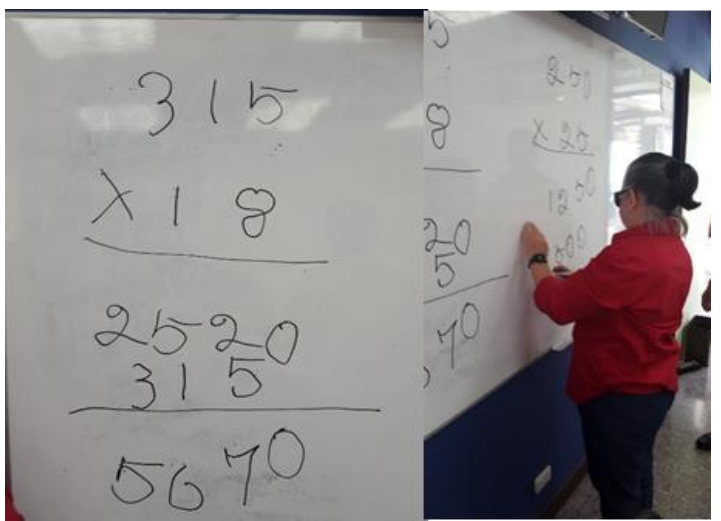

Figura 2. Desarrollo de operaciones básicas en la pizarra

Como se puede ver, la ubicación de los numerales no es el correcto, aspecto que tiene que debe quedar muy claro a los niños cuando están estudiando este tema. Es importante señalar, que se determinó que el problema de la estudiante no es de contenidos sino del uso del recurso didáctico (la pizarra).

\section{Resultados y Discusión}

En el entorno virtual de la asignatura de Matemática I para I y II Ciclos se propicia la educación inclusiva y para ello los estudiantes cuentan con material de apoyo que les permite reforzar los contenidos aprendidos y realizar los trabajos académicos. Por otro lado, hay que señalar que además de lo que se estipula en la Misión de la UNED. La Universidad Estatal a Distancia (UNED) ... Su misión es ofrecer educación superior a todos los sectores de la población, especialmente a aquellos que por razones económicas, sociales, geográficas, culturales, etarias, de discapacidad o de género, requieren oportunidades para una inserción real y equitativa en la sociedad. (UNED, 2013) 
también existe un compromiso y convencimiento de que la educación en la UNED tiene que ser inclusiva, donde todos tengan cabida y posibilidades de éxito en sus estudios.

Ahora bien, antes de implementar los cambios en la entrega de la docencia para esta estudiante, se consideró la definición de objeto de aprendizaje de Aguilera \& Bejarano (2013) y sus características donde los objetos de aprendizaje no son otra cosa que unidades de aprendizaje que pueden ser actualizadas cuando el docente lo considere conveniente de acuerdo con las necesidades e intereses de los estudiantes. Estos objetos de aprendizaje pueden estar en cualquier formato de salida, físico o digital y por lo general se pueden utilizar en cualquier plataforma LMS. (Learning management system o sistema de gestión para la enseñanza). Estos mismos autores, en relación con las características de los objetos de aprendizaje las clasifica en tres dimensiones: pedagógica porque persiguen objetivos relacionados con el proceso de aprendizaje, tecnológica que tiene que ver propiamente con las características del software con que fueron construidos (portabilidad, compatibilidad) y la interactividad del usuario con la computadora, esto es, con contenidos y actividades que motiven al estudiante a utilizar el objeto de estudio. Para la elaboración de la estrategia metodológica se utilizó la plataforma educativa virtual Moodle ya que el objeto de aprendizaje diseñado para la estudiante funciona perfectamente con todas las herramientas que posee este entorno y además el formato de salida de los materiales están adecuados para ella. Se utilizó la herramienta foro para que la estudiante participara al igual que sus compañeros de las actividades programadas durante el cuatrimestre. Por otro lado, la alumna asiste a la tutoría presencial con el resto de sus compañeros, y el tutor encargado de impartir la tutoría, tiene mucha experiencia en el trabajo con estudiantes con discapacidad visual.

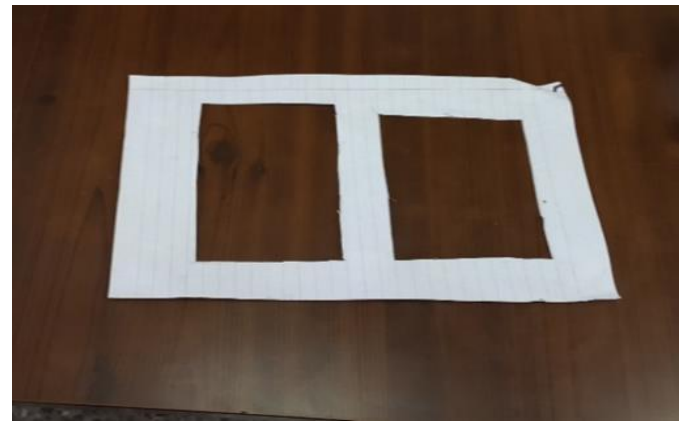

Figura 3. Adecuación del material. Fuente: Fotografías tomadas el día de atención. Respaldos de la Cátedra de Matemáticas Básicas. Figura 3. Adecuación del material. Fuente: Fotografías tomadas el día de atención. Respaldos de la Cátedra de Matemáticas Básicas.
En cuanto a la adecuación del uso de la pizarra, se inició con la propuesta de hacer una plantilla con "rectángulos huecos" que le permitieran establecer por medio del tacto, los espacios que debía utilizar para escribir los numerales como se muestra en la figura 3.

No obstante, como se utilizaron hojas de cuaderno, se puedo observar que el material no era lo suficientemente resistente, por lo que se utilizó cartulinas para hacer el material más fuerte y resistente al tacto de la estudiante (Figura 4).

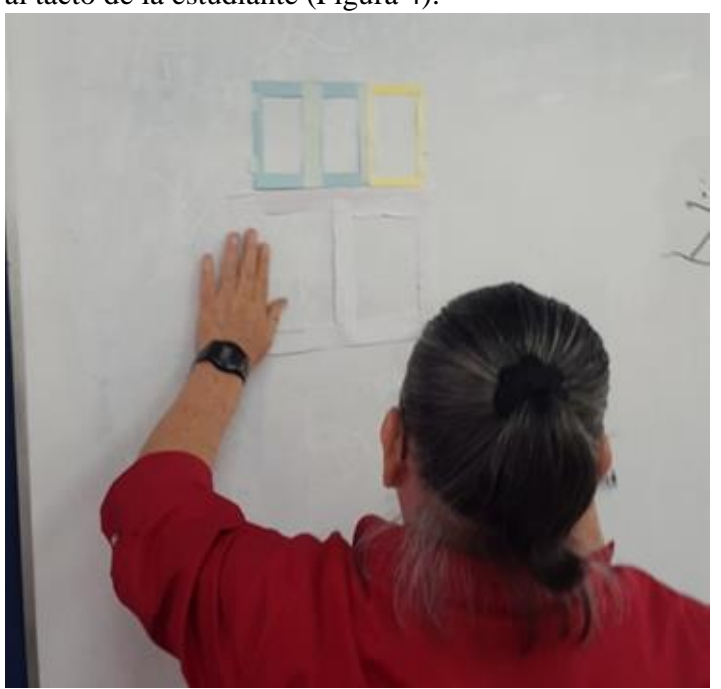

Figura 4. Uso del material adecuado. Fuente: Fotografías tomadas el día de atención. Respaldos de la Cátedra de Matemáticas Básicas con la autorización de la estudiante.

Se observó también que el material podía ser útil para el abordaje del tema en el desarrollo de la mini-clase, pero que era útil para realizar la ubicación de las cifras, pero no era funcional para colocar una operación con más de dos cifras como se muestra en la figura 5.

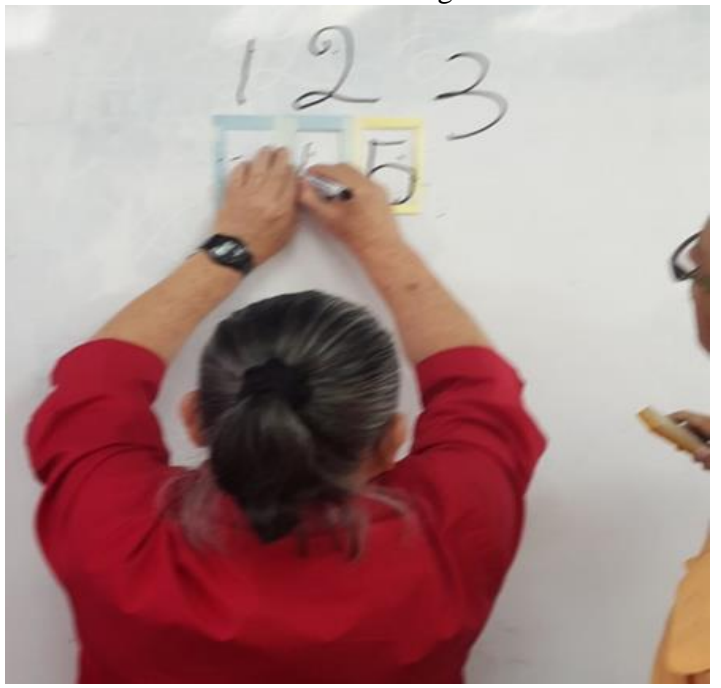

Figura 5. Limitaciones en el uso del material. Fuente: Fotografías tomadas el día de atención. Respaldos de la Cátedra de Matemáticas Básicas con la autorización de la estudiante. 
Por esta razón, con la ayuda de una regla, cartulina, lápiz y una cortadora, se realizó una nueva adecuación del material, de manera que el nuevo instrumento sirviera de plantilla como un cuadro de valores posicional para efectuar operaciones aritméticas como se muestra en la figura 6.

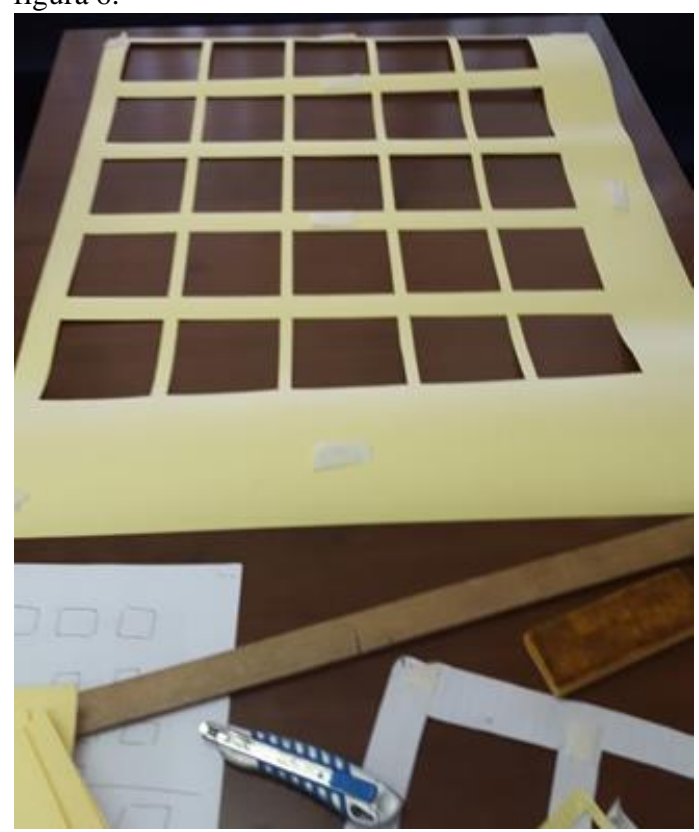

Figura 6. Plantilla. Ampliación de la herramienta. Fuente: Fotografías tomadas el día de atención. Respaldos de la Cátedra de Matemáticas Básicas

Cuando la estudiante hizo uso del recurso, se logró observar una gran mejoría en el desempeño y el uso espacial de la pizarra, lo cual le brindó confianza y seguridad como docente para desarrollar cualquier tipo de multiplicación, lo que le permitió llevar a cabo su mini-clase sin ningún problema.

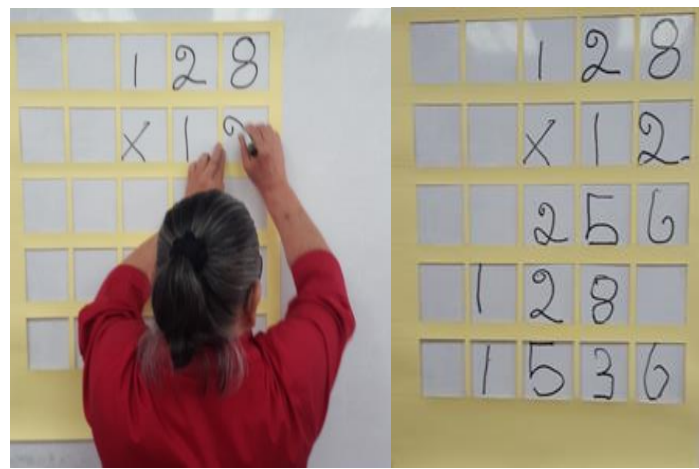

Figura 7. Uso de la ampliación en la herramienta. Fuente: Fotografías tomadas el día de atención. Respaldos de la Cátedra de Matemáticas Básicas con la autorización de la estudiante

\section{Conclusiones}

Es indispensable incorporar los principios y pautas del Diseño Universal para el Aprendizaje (DUA), en todo el quehacer académico y administrativo de la UNED, y de esta manera asegurarles no solo el éxito a los estudiantes en su trabajo escolar, sino que también en los espacios deportivos, culturales, de extensión, vida estudiantil, entre otros, para que puedan expresarse libremente independientemente de la diversidad de cada uno de ellos.

También, es importante cuando se diseñe una estrategia metodológica tener presente el segundo principio del DUA: proporcionar múltiples formas de representación y con ello considerar diferentes opciones que tengan que ver con la salida de la información auditiva, visual, el uso del lenguaje y la simbología matemática, así como considerar los conocimientos previos, maximizar el uso de la memoria y el uso de patrones para las ideas principales y la relación entre ellos.

De igual manera, con este tipo de población es imprescindible propiciar diferentes formas de comunicación, apoyarlos en la planificación y el desarrollo de estrategias y estimular en ellos el seguimiento de sus avances en su trabajo académico.

Por otro lado, hay que tener presente en el diseño de cualquier estrategia metodológica la primera pauta del DUA: usar múltiples formas de presentación, y la tercera que tiene que ver con el uso de las múltiples formas de motivación, por lo que es necesario proporcionarles diferentes formas de búsqueda de sus intereses que mejoren su autoestima y les permita enfrentar nuevos desafíos tanto en su vida académica como personal.

También hay que tener claro que en un sistema a distancia como el de la UNED, el que enseña no es el tutor, sino que toda la institución con sus distintas dependencias y tecnología, quien organizacionalmente asegura que el acto de aprendizaje se lleve a cabo de la mejor manera, de ahí entonces la necesidad de trabajar en forma conjunta y coordinada para atender a este tipo de población.

Finalmente, es necesario tener presente que como académicos de la UNED estamos en la obligación de formar docentes comprometidos con una educación más integral y humana, reflexivos y respetuosos de la diversidad que nos permita entre todos, construir una educación inclusiva articulando la teoría con la práctica. 


\section{Referencias}

Aguilera \& Bejarano. (2013). Características deseables en los objetos de aprendizaje para el estudio de estructuras de control aplicadas a la lógica de la programación. Recuperado de https://www.uned.ac.cr/academica/edutec/me moria/ponencias/roy_gabriela_111.pdf

Badilla, J. E. (2016). Fracciones con legos. Material no publicado.

Gutiérrez \& Prieto. (2002). Mediación Pedagógica. Guatemala: Colección Programa EDUSAC.

Ministerio de Educación Nacional (MEP). (2006). Orientaciones Pedagógicas para la atención educativa a estudiantes con limitación visual. Recuperado de http://www.colombiaaprende.edu.co/html/mic rositios/1752/articles-320691_archivo_2.pdf. [Consultado 11 jun, 2017].

Ministerio de Educación Nacional (MEP). (2012). Programas de Estudio de Matemáticas. San José, Costa Rica: Imprenta Nacional. 